\title{
The Bloch transform and applications
}

\author{
Grégoire Allaire*, Carlos Conca ${ }^{\dagger}$ \\ and \\ Muthusamy Vanninathan ${ }^{\ddagger}$
}

\begin{abstract}
The Fourier Transform is one of the basic concepts in the mathematical analysis of partial differential equations in Physics. As is well-known, one of its most curious properties is the fact that it converts derivatives into multiplications. This makes it a very useful tool, as it transforms differential equations into algebraic ones. Naturally, it also has its limits. Particularly, it is not evident that it can be applied to differential equations with variable coefficients, that is, in the treatment of heterogeneous media.

In this Lecture, we show one possible way to generalize the ideas of Fourier Analysis, so as to make them accessible to the study of non-homogeneous periodic media. We would like to generate a new technique, oriented to understanding certain mathematical phenomena in Homogenization Theory. To do so, we use a special class of functions, known as Bloch waves, which are commonly used in Solid State Physics. The resulting methodology is illustrated through two applications. We see how it applies in the classical problem of homogenization of elliptic operators in arbitrary domains of $\mathbb{R}^{N}$ with periodically oscillating coefficients. We also use it to study the asymptotic behaviour of the spectrum of some periodic structures, and more precisely, we consider it in the context of the wave equation in a bounded periodic heterogeneous medium.

This Lecture reviews and unifies recent joint works of the authors [3], [6], [7], [14].

KEYWORDS : homogenization, periodic structures, Bloch waves, spectral analysis, wave equation.

AMS Classification : 35A25, 35B20, 42C30.
\end{abstract}

\section{Introduction}

Our starting point is the spectral analysis of the operator $(-\Delta)$ in $\mathbb{R}^{N}$ which, as usual, is considered as an unbounded operator acting in $L^{2}\left(\mathbb{R}^{N}\right)$ and domain $D(-\Delta)=H^{2}\left(\mathbb{R}^{N}\right)$. It is well-known that the spectrum of this operator consists of the non-negative real axis and that the plane waves $e^{i \eta \cdot y}$ with $|\eta|^{2}=\lambda$ can be considered as "generalized eigenfunctions" with "eigenvalue" $\lambda \geq 0$. These functions are not elements of $L^{2}\left(\mathbb{R}^{N}\right)$ but they span all of $L^{2}\left(\mathbb{R}^{N}\right)$, since they provide the spectral resolution of the identity in the sense of Fourier inversion:

$$
f(y)=\frac{1}{(2 \pi)^{N / 2}} \int_{\mathbb{R}^{N}} \hat{f}(\eta) e^{i \eta \cdot y} d \eta
$$

\footnotetext{
${ }^{*}$ Commissariat à l'Energie Atomique, DRN/DMT/SERMA, C.E. Saclay, 91191 Gif sur Yvette, FRANCE and Laboratoire d'Analyse Numérique, Université Pierre et Marie Curie.

${ }^{\dagger}$ Departamento de Ingenierí́a Matemática, Universidad de Chile, Casilla 170/3 - Correo 3, Santiago, CHILE.

${ }^{\ddagger}$ Tata Institute of Fundamental Research Center, IISc-TIFR Mathematics Programme, P.O. Box 1234, Bangalore 560012, INDIA.
} 
(here, $\hat{f}$ denotes the classical Fourier-Plancherel transform of $f$ ). Indeed, by means of planes waves and Fourier analysis one can derive the full spectral resolution of $(-\Delta)$ (see e.g. [12], pp. 60-61).

In this example, the medium does not vary at all since it is represented by a differential operator with constant coefficients. In contrast with this case, we are going to analyze media which oscillate rapidly, i.e., the case where the coefficients representing the medium have a small period. More precisely, let us consider the operator

$$
\mathcal{A}=-\frac{\partial}{\partial y_{k}}\left(a_{k \ell}(y) \frac{\partial}{\partial y_{\ell}}\right)
$$

where the coefficients $a_{k \ell}$ are assumed to satisfy

$$
\left\{\begin{array}{l}
a_{k \ell} \in L_{\#}^{\infty}(Y), \text { where } Y=\left[0,2 \pi\left[{ }^{N}, \text { i.e., each } a_{k \ell}\right. \text { is a }\right. \\
Y \text {-periodic bounded measurable function defined on } \mathbb{R}^{N}, \\
\exists \alpha>0 \quad \text { such that } a_{k \ell}(y) \xi_{k} \xi_{\ell} \geq \alpha|\xi|^{2} \quad \text { (ellipticity), } \\
a_{k \ell}=a_{\ell k} \quad \forall k, l=1, \ldots, N \quad \text { (symmetry). }
\end{array}\right.
$$

(As usual, summation with respect to the repeated indices is understood throughout this paper, and the constants appearing in various estimates independent of $\varepsilon$ are generically denoted by $c$ ).

The functions that will now play the role of generalized eigenfunctions are known as (classical) Bloch waves. These waves were originally introduced in Solid State Physics in the context of propagation of electrons in a crystal, see [9]. Several questions and properties of periodic media can be translated in terms of Bloch waves. The first mathematical result in this topic is due to I.M. Gelfand [19]. In his paper, a proof is outlined of the so-called Parseval's Identity for functions in $L^{2}\left(\mathbb{R}^{N}\right)$ (see below, Theorem 1.1). The reader may refer to the book [12] for a wide variety of applications in the vibrations of fluid-solid structures and to the works of Allaire and Conca [3], [4], [5] who have succeeded in using these waves to study the asymptotic behaviour of the spectrum of some periodic structures. Additional references on Bloch waves are [8], [27], [31], [33], [39].

We are interested in the spectral resolution of $\mathcal{A}$ in $L^{2}\left(\mathbb{R}^{N}\right)$. For this purpose, the classical method of Bloch is used and it consists of introducing a family of spectral problems parameterized by $\eta \in \mathbb{R}^{N}$ : Find $\lambda=\lambda(\eta) \in \mathbb{R}$ and $\psi=\psi(y ; \eta)$ (not identically zero) such that

$$
\left\{\begin{array}{l}
\mathcal{A} \psi(\cdot ; \eta)=\lambda \psi(\cdot ; \eta) \text { in } \mathbb{R}^{N}, \\
\psi(\cdot ; \eta) \text { is }(\eta, Y) \text {-periodic, i.e., } \\
\psi(y+2 \pi m ; \eta)=e^{2 \pi i m \text { cdot } \eta} \psi(y) \quad \forall m \in \mathbb{Z}^{N}, y \in \mathbb{R}^{N}
\end{array}\right.
$$

First of all, it is clear that the above problem remains the same if $\eta$ is replaced by $\eta+m$, $m \in \mathbb{Z}^{N}$. So, there is no loss of generality in confining $\eta$ to the cell $Y^{\prime}=\left[0,1{ }^{N}\right.$. (In what follows, we shall take $\eta$ in the translated cell $Y^{\prime}=\left[-\frac{1}{2}, \frac{1}{2}\left[{ }^{N}\right.\right.$, again without loss of generality). We refer to $Y^{\prime}$ as the reciprocal cell of $Y$ (In the Physics literature, $Y^{\prime}$ is known as the first Brillouin zone). Solutions $\psi$ of (3) are usually called Bloch waves or Bloch eigenvectors; they can be motivated in a couple of ways, see [12] or [14]. As already mentioned, if the medium were homogeneous, then it is classical to use plane waves $e^{i \eta \cdot y}$ to solve the problem. Bloch waves are natural generalizations of plane waves to treat periodic media.

Periodic media in one dimension were studied by Floquet [18] prior to Bloch. Following his ansatz, we look for solutions of (3) which are products of $Y$-periodic functions with solutions in the homogenized media, i.e., plane waves:

$$
\psi(y ; \eta)=e^{i \eta c \operatorname{dot} y} \phi(y ; \eta), \quad \phi(\cdot ; \eta) \text { is } Y \text {-periodic. }
$$


This transformation maps (3) into a new problem where the parameter $\eta$ appears in the operator rather than in the boundary condition: Find $\lambda=\lambda(\eta) \in \mathbb{R}$ and $\phi=\phi(y ; \eta)$ (not identically zero) such that

$$
\left\{\begin{array}{l}
\mathcal{A}(\eta) \phi=\lambda \phi \text { in } \mathbb{R}^{N} \\
\phi \text { is } Y \text {-periodic. }
\end{array}\right.
$$

Here the operator $\mathcal{A}(\eta)$ is defined by

$$
\mathcal{A}(\eta) \stackrel{\text { def }}{=}-\left(\frac{\partial}{\partial y_{k}}+i \eta_{k}\right)\left(a_{k \ell}(y)\left(\frac{\partial}{\partial y_{\ell}}+i \eta_{\ell}\right)\right)
$$

and it is referred to in the literature as the shifted operator.

It is well-known that, due to ellipticity and symmetry hypothesis, the above problem (5) admits a sequence of eigenvalues and eigenvectors (which are also referred to as Bloch eigenvalues and Bloch eigenvectors or Bloch waves, respectively) with the following properties:

$$
\left\{\begin{array}{l}
\lambda_{1}(\eta) \leq \cdots \leq \lambda_{m}(\eta) \leq \cdots \rightarrow \infty \\
\left\{\phi_{m}(\cdot ; \eta)\right\}_{m=1}^{\infty} \text { forms an orthonormal basis in } L_{\#}^{2}(Y) \\
\forall m \geq 1, \lambda_{m}(\eta) \text { defines a Lipschitz continuous function of } \eta \text { in } Y^{\prime}
\end{array}\right.
$$

Thanks to the above parameterized family of eigenvalues, one can completely describe the spectral resolution of $\mathcal{A}$ as an unbounded, self-adjoint operator in $L^{2}\left(\mathbb{R}^{N}\right)$. Roughly speaking, the results are as follows: The spectrum of $\mathcal{A}$, denoted $\sigma(\mathcal{A})$, has a band structure and more exactly, it coincides with the so-called Bloch spectrum which is defined as the union of the images of all the mappings $\lambda_{m}(\cdot)$, i.e.,

$$
\sigma(\mathcal{A})=\sigma_{\text {Bloch }} \stackrel{\text { def }}{=} \bigcup_{m=1}^{\infty}\left[\min _{\eta \in Y^{\prime}} \lambda_{m}(\eta), \max _{\eta \in Y^{\prime}} \lambda_{m}(\eta)\right]
$$

(It can be proved in some examples that the gaps in $\sigma_{\text {Bloch }}$ are not empty, see [17]). The family

$$
\left\{e^{i \eta \cdot y} \phi_{m}(y ; \eta) \mid m \geq 1, \eta \in Y^{\prime}\right\}
$$

forms a basis of $L^{2}\left(\mathbb{R}^{N}\right)$ in a generalized sense, and $L^{2}\left(\mathbb{R}^{N}\right)$ can be identified with $L^{2}\left(Y^{\prime}, \ell^{2}(\mathbb{N})\right)$ via Parseval's Identity. This is the essence of how the spectral family of $\mathcal{A}$ can be constructed (see [12]), and also the foundation of the following result, a proof of which can be found in [8] or [12]:

Theorem 1.1. Let $g \in L^{2}\left(\mathbb{R}_{y}^{N}\right)$. The $m^{\text {th }}$ Bloch coefficient of $g$ is defined as follows:

$$
\hat{g}_{m}(\eta)=\int_{\mathbb{R}^{N}} g(y) e^{-i \eta \cdot y} \bar{\phi}_{m}(y ; \eta) d y \quad \forall m \geq 1, \eta \in Y^{\prime}
$$

Then the following inverse formula holds:

$$
g(y)=\int_{Y^{\prime}} \sum_{m=1}^{\infty} \hat{g}_{m}(\eta) e^{i \eta \cdot y} \phi_{m}(y ; \eta) d \eta
$$

Further, we have Parseval's Identity:

$$
\int_{\mathbb{R}^{N}}|g(y)|^{2} d y=\int_{Y^{\prime}} \sum_{m=1}^{\infty}\left|\hat{g}_{m}(\eta)\right|^{2} d \eta .
$$




\subsection{Classical periodic homogenization}

As a first application of the Bloch decomposition method, we will show how one can reestablish some of the classical results of the periodic homogenization theory in arbitrary domains. The principal result is stated in Theorem 1.2. Although it is not new, the method sheds new light and offers a non-traditional way of calculating the homogenized coefficients. There are many ways to obtain these coefficients and there is a vast body of work in the literature which justifies the limiting procedure. The basic reference is the book [8] which presents an application of the method of multiple scale expansion to homogenization and this technique seems to be the easiest way to obtain the homogenized medium. Justification of this method is usually done by Tartar's method which he developed in large part in association with F. Murat, see [25], [36]. Their method is very general and it goes beyond the case of periodically oscillating coefficients. However, if the medium is periodic, there is an alternative procedure to pass to the limit by using the notion of two-scale weak convergence introduced in [2] and [26].

Here we follow a different approach based on Fourier analysis and introduced in [14]. For each $\varepsilon>0$, let us consider the operator $\mathcal{A}^{\varepsilon}$, where

$$
\mathcal{A}^{\varepsilon} \stackrel{\text { def }}{=}-\frac{\partial}{\partial x_{k}}\left(a_{k \ell}^{\varepsilon}(x) \frac{\partial}{\partial x_{\ell}}\right) \quad \text { with } \quad a_{k \ell}^{\varepsilon}(x)=a_{k \ell}\left(\frac{x}{\varepsilon}\right) .
$$

From the theory of homogenization, it is known that there is a corresponding homogenized operator $\mathcal{A}^{*}$ given by

$$
\mathcal{A}^{*} \stackrel{\text { def }}{=}-\frac{\partial}{\partial x_{k}}\left(q_{k \ell} \frac{\partial}{\partial x_{\ell}}\right) \text {. }
$$

The homogenized coefficients $q_{k \ell}$ are constants and their definition can be found, e.g., in [8] p. 17; we will recall it later (see (19)). It is known that $\left(q_{k \ell}\right)$ is a symmetric, positive definite matrix: $q_{k \ell} \xi_{k} \xi_{\ell} \geq \alpha|\xi|^{2} \forall \xi \in \mathbb{R}^{N}$, where $\alpha>0$ is the same constant appearing in (2).

Our main result regarding this subject is:

Theorem 1.2. Let $\Omega$ be an arbitrary domain in $\mathbb{R}^{N}$. Let the coefficients $a_{k \ell}$ satisfy assumptions (2). Suppose $\left\{u^{\varepsilon}\right\}$ is a sequence in $H^{1}(\Omega)$ and $u^{*} \in H^{1}(\Omega), f \in L^{2}(\Omega)$ are such that

$$
\left\{\begin{array}{l}
u^{\varepsilon} \rightarrow u^{*} \text { in } H^{1}(\Omega) \text {-weakly } \\
\mathcal{A}^{\varepsilon} u^{\varepsilon}=f \quad \text { in } \Omega
\end{array}\right.
$$

Then the stress vector $\sigma_{k}^{\varepsilon} \stackrel{\text { def }}{=} a_{k \ell}^{\varepsilon} \frac{\partial u^{\varepsilon}}{\partial x_{\ell}}$ converges weakly in $L^{2}(\Omega)$ to the corresponding homogenized stress vector:

$$
\sigma_{k}^{\varepsilon} \rightarrow q_{k \ell} \frac{\partial u^{*}}{\partial x_{\ell}} \quad \text { in } \quad L^{2}(\Omega) \text {-weakly } \quad \forall k=1, \ldots, N .
$$

In particular, $u^{*}$ satisfies the homogenized equation, namely

$$
\mathcal{A}^{*} u^{*}=f \quad \text { in } \Omega \text {. }
$$

In the above theorem, we have assumed the weak convergence of $u^{\varepsilon}$. This is because a $H^{1}$ bound on $u^{\varepsilon}$ is not guaranteed. However, if $\Omega$ is a bounded domain and $u^{\varepsilon}$ satisfies certain boundary conditions (e.g. Dirichlet) on the boundary $\partial \Omega$ in addition to the equation $\mathcal{A}^{\varepsilon} u^{\varepsilon}=f$ in $\Omega$ such a priori estimate on $u^{\varepsilon}$ will be a consequence of ellipticity and Poincaré inequality. In case $\Omega$ is unbounded, say $\Omega=\mathbb{R}^{N}$, then we do not have an estimate on $u^{\varepsilon}$ in $H^{1}\left(\mathbb{R}^{N}\right)$. However, if we consider $\mathcal{A}^{\varepsilon}+I$ instead of $\mathcal{A}^{\varepsilon}$, then the bound in $H^{1}\left(\mathbb{R}^{N}\right)$ is automatic. In these cases, we would be able to deduce the usual homogenization results. For the sake of completeness, we announce them separately. 
Corollary 1.3. Let $\Omega$ be a bounded domain in $\mathbb{R}^{N}$. Let $\left(a_{k \ell}\right)$ and $f$ be as in Theorem 1.2. Consider $v^{\varepsilon}$ the unique solution of

$$
\mathcal{A}^{\varepsilon} v^{\varepsilon}=f \quad \text { in } \quad \Omega, \quad v^{\varepsilon} \in H_{0}^{1}(\Omega) .
$$

Then

$$
\begin{aligned}
v^{\varepsilon} & \rightarrow v^{*} \text { in } \quad H_{0}^{1}(\Omega) \text {-weakly, } \\
a_{k \ell}^{\varepsilon} \frac{\partial v^{\varepsilon}}{\partial x_{\ell}} & \rightarrow q_{k \ell} \frac{\partial v^{*}}{\partial x_{\ell}} \quad \text { in } \quad L^{2}(\Omega) \text {-weakly } \quad \forall k=1, \ldots, N,
\end{aligned}
$$

where $v^{*}$ is the unique solution satisfying

$$
\mathcal{A}^{*} v^{*}=f \quad \text { in } \Omega, \quad v^{*} \in H_{0}^{1}(\Omega) \text {. }
$$

Corollary 1.4. Let $\left(a_{k \ell}\right)$ and $f$ be as in Theorem 1.2. Consider $w^{\varepsilon}$ the unique solution of

$$
\mathcal{A}^{\varepsilon} w^{\varepsilon}+w^{\varepsilon}=f \quad \text { in } \quad \mathbb{R}^{N}, \quad w^{\varepsilon} \in H^{1}\left(\mathbb{R}^{N}\right) .
$$

Then

$$
\begin{aligned}
w^{\varepsilon} & \rightarrow w^{*} \text { in } H^{1}\left(\mathbb{R}^{N}\right) \text {-weakly, } \\
a_{k \ell}^{\varepsilon} \frac{\partial w^{\varepsilon}}{\partial x_{\ell}} & \rightarrow q_{k \ell} \frac{\partial w^{*}}{\partial x_{\ell}} \quad \text { in } \quad L^{2}\left(\mathbb{R}^{N}\right) \text {-weakly } \quad \forall k=1, \ldots, N,
\end{aligned}
$$

where $w^{*}$ is the unique solution of

$$
\mathcal{A}^{*} w^{*}+w^{*}=f \quad \text { in } \quad \mathbb{R}^{N}, \quad w^{*} \in H^{1}\left(\mathbb{R}^{N}\right) .
$$

L. Tartar proved these results using his method of homogenization and a proof is available in [8], see also [32]. His proof handles even non-symmetric coefficients $\left(a_{k \ell}\right)$. We are going to re-prove Theorem 1.2 using the so-called Bloch-wave method. Even though we treat here only the case of symmetric coefficients, our method has recently been enlarged to cover the case of non-symmetric coefficients. We thus recover fully Tartar's theorem (see [37]). Since this is a spectral method, we are naturally led to suppose the symmetry of the coefficients $\left(a_{k \ell}\right)$. The method, a version of which appeared in [34], works in the following way: the original problem is first transformed into a set of algebraic equations in the Bloch space. Next, it is shown that all Bloch harmonics corresponding to $m \geq 2$ can be neglected in the homogenization process. This explains why oscillations present in the solution are not well approximated by the homogenized one. Finally, we pass to the limit as $\varepsilon$ tends to zero in the first harmonic and establish that the Bloch waves representing the periodic medium approach Fourier waves representing the homogenized medium. The latter step is quite straightforward if we work in the entire space (in the case of an arbitrary domain, localization is involved and this complicates the analysis a little bit), but it demands certain regularity of the dominant Bloch eigenvalue near the origin. This is a technical result which is proven in [14], §2. After passage to the limit, the limiting equation is easily interpreted as a result of homogenization in the Fourier space. To conclude this paragraph regarding Fourier analytic approaches to classical homogenization problems, let us mention the work by R. Morgan and I. Babuška [24].

\subsection{Spectral asymptotic analysis}

In our second application, we show how the Bloch wave method can be used to study the asymptotic behaviour of the spectrum of some periodic structures. To simplify the exposition, we present this method in the context of the wave equation in a bounded periodic 
heterogeneous medium. We study the asymptotic behaviour of its spectrum as the structure period goes to zero. As is easily inferred, the physical motivation of such a study is to better understand wave propagation in periodic media, and it is well-known that this problem often relies on the analysis of the vibrations modes of the medium. The wave equation is just a model, in truth, our original motivation comes from more complicated models describing the vibrations of fluid-solid structures. These more physical models were proposed by J. Planchard in the early 80's (see e.g. [16], or [29], [30]) and extensively studied using the Bloch-wave method in [4], [5], [12], [13], [15].

To review part of these works, let us consider an arbitrary bounded domain $\Omega$ in $\mathbb{R}^{N}$ and, for each $\varepsilon>0$, let $\mathcal{A}^{\varepsilon}$ be the operator defined by (8). Associated with $\mathcal{A}^{\varepsilon}$ we consider the following spectral problem for the wave equation in $\Omega$ : Find all couples $\left(\lambda^{\varepsilon}, v^{\varepsilon}\right) \in \mathbb{R} \times H_{0}^{1}(\Omega)$, $v^{\varepsilon} \not \equiv 0$, such that

$$
\mathcal{A}^{\varepsilon} v^{\varepsilon}=\lambda^{\varepsilon} v^{\varepsilon} \quad \text { in } \quad \Omega, \quad v^{\varepsilon} \in H_{0}^{1}(\Omega)
$$

At this point, allow us a word of warning regarding the notation. In the articles just quoted, instead of working on the eigenvalue problem associated with $\mathcal{A}^{\varepsilon}$, as we do here, work is done on the eigenvalues of $\left(\mathcal{A}^{\varepsilon}\right)^{-1}$. The only consequence of this change of convention is that the eigen-variable $\lambda^{\varepsilon}$ in (10) corresponds in these references to $\frac{1}{\lambda^{\varepsilon}}$, but of course, results in both cases are equivalent, qualitatively.

With that in mind, let us denote by $\sigma^{\varepsilon}$ the set of eigenvalues of (10). As is well-known, $\sigma^{\varepsilon}$ is made of a countable sequence of eigenvalues which converge to $+\infty$ and each of them is of finite multiplicity, i.e.,

$$
\sigma^{\varepsilon}=\bigcup_{m \geq 1}\left\{\lambda_{m}^{\varepsilon}\right\} \quad \text { with } \quad 0<\lambda_{1}^{\varepsilon} \leq \cdots \leq \lambda_{m}^{\varepsilon} \leq \cdots \rightarrow+\infty
$$

To each $\lambda_{m}^{\epsilon}$ is associated a normalized eigenfunction $v_{m}^{\epsilon} \in L^{2}(\Omega)$ such that $\left\|v_{m}^{\varepsilon}\right\|_{L^{2}(\Omega)}=1$, and the family $\left\{v_{m}^{\varepsilon}\right\}_{m}$ is an orthonormal basis of $L^{2}(\Omega)$. It is further well-known that, as the period $\varepsilon$ tends to zero, $\sigma^{\varepsilon}$ converges to the spectrum $\sigma^{*}$ of the homogenized problem (see e.g. [10], [21], [28], [38]):

$$
\mathcal{A}^{*} v=\lambda v \quad \text { in } \Omega, \text { quad } v \in H_{0}^{1}(\Omega),
$$

where $\mathcal{A}^{*}$ is the homogenized operator defined in (9). This is, for all $m \geq 1$,

$$
\lambda_{m}^{\varepsilon} \rightarrow \lambda_{m}
$$

and the corresponding eigenfunction $v_{m}^{\varepsilon}$ converges strongly to $v_{m}$ in $L^{2}(\Omega)$, as $\varepsilon \rightarrow 0$. Here, $\lambda_{m}$ is the $m^{t h}$ eigenvalue of the homogenized problem associated with $v_{m}$. Recall that $\mathcal{A}^{*}$ is strictly elliptic (with the same constant appearing in (2)) and that therefore has a compact inverse in $L^{2}(\Omega)$ (see e.g. [8]). Its spectrum, denoted $\sigma^{*}$, is also made of a countable sequence of eigenvalues of finite multiplicity.

Although it seems comprehensive, this convergence result does not completely describe the asymptotic behaviour of all the sequences of eigenvalues $\lambda^{\varepsilon}$ that converge to $+\infty$. In particular, letting $\varepsilon \rightarrow 0$ and $m \rightarrow+\infty$ it is possible to construct sequences of eigenvalues $\lambda_{m(\varepsilon)}^{\epsilon}$ which converge to $+\infty$ and whose corresponding eigenfunctions do not converge strongly in $L^{2}(\Omega)$. In fact, they converge to 0 weakly in $L^{2}(\Omega)$ (see [3], or [4] for a similar case). It is therefore interesting to consider suitable renormalizations of the set $\sigma^{\varepsilon}$ and study their asymptotic behaviour. More exactly, our goal in the remainder part of this section is to characterize the renormalized limits of the spectrum $\lim _{\varepsilon \rightarrow 0} \varepsilon^{\alpha} \sigma^{\varepsilon}$ where $\alpha$ is a positive number. This situation is called a low frequency limit, while the classical convergence result (12) and the strong convergence of the corresponding eigenvectors gives a high frequency limit (It is also called macroscopic limit). 
Let us first consider the case of eigenvalues of the order of $\varepsilon^{-2}(\alpha=2)$ that, as we will see, correspond to a critical size in the sense that the asymptotic behaviour of $\varepsilon^{2} \sigma^{\varepsilon}$ is particular regarding the case $\alpha \neq 2$. First of all, let us begin by pinpointing the definition of this limit set: By $\lim _{\varepsilon \rightarrow 0} \varepsilon^{2} \sigma^{\varepsilon}$ we mean the following set of cluster points:

$$
\lim _{\varepsilon \rightarrow 0} \varepsilon^{2} \sigma^{\varepsilon}=\left\{\lambda \in \mathbb{R}^{+} \mid \begin{array}{l}
\exists \text { a subsequence, still denoted by } \varepsilon \text { and } \exists\left(\lambda^{\varepsilon}, v^{\varepsilon}\right), \\
\text { solutions of }(10), \text { such that } \varepsilon^{2} \lambda^{\varepsilon} \rightarrow \lambda
\end{array}\right\} .
$$

The main characterization result for this renormalized limit makes use of the Bloch spectrum, that we define in (7) using the Bloch waves and the Bloch eigenvalues. To announce this result, we also need to define a so-called boundary layer spectrum $\sigma_{\text {boundary }}$. Let us consider a sequence of eigenvalues and eigenvectors $\left(\lambda^{\varepsilon}, v^{\varepsilon}\right)$ solutions of (10). If, for a subsequence still denoted by $\varepsilon$, there exists a limit $\lambda$ such that

$$
\left\{\begin{array}{l}
\left\|v^{\varepsilon}\right\|_{L^{2}(\Omega)}=1 \quad \text { and } \quad \lim _{\varepsilon \rightarrow 0} \varepsilon^{2} \lambda^{\varepsilon}=\lambda \\
\lim _{\varepsilon \rightarrow 0}\left\|v^{\varepsilon}\right\|_{L^{2}(\omega)}=0
\end{array}\right.
$$

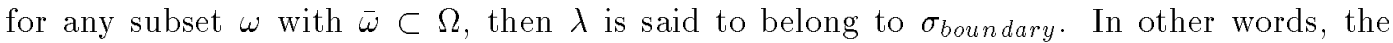
boundary layer spectrum is defined by

$$
\sigma_{\text {boundary }}=\left\{\lambda \in \mathbb{R}^{+} \mid \exists\left(\lambda^{\varepsilon}, v^{\varepsilon}\right) \text { solutions of (10) satisfying (13) }\right\} .
$$

From a physical point of view, $\sigma_{\text {boundary }}$ corresponds to sequences of eigenvectors concentrating near the boundary of $\Omega$. Comparing its definition with that of the macroscopic spectrum $\sigma_{\text {Bloch }}$, we see that $\sigma_{\text {boundary }}$ may depend on the choice of the sequence $\epsilon$.

Our main result (announced in [6], [7]) is

\section{Theorem 1.5.}

$$
\lim _{\varepsilon \rightarrow 0} \varepsilon^{2} \sigma^{\varepsilon}=\sigma_{\text {boundary }} \cup \sigma_{\text {Bloch }} .
$$

The proof of Theorem 1.5 is sketched in $\S 3$. It relies on a notion of Bloch limiting measures which play, more or less, the role of semi-classical (or Wigner) measures in the context of Schrödinger equation (see e.g. [20], [22], [23], [35]). Here, as already mentioned, the scaling $\varepsilon^{-2}$ of the eigenvalues can be interpreted as a critical size. Indeed, for any other scaling, we find a simpler result since there is no interaction between the period size $\varepsilon$ and the frequency size:

Theorem 1.6. Let $a_{\varepsilon} \in \mathbb{R}^{+}$be a sequence converging to 0 with $\varepsilon$ and such that, either

$$
\lim _{\varepsilon \rightarrow 0} \frac{a_{\varepsilon}}{\varepsilon}=0, \quad \text { or } \quad \lim _{\varepsilon \rightarrow 0} \frac{a_{\varepsilon}}{\varepsilon}=+\infty .
$$

Then,

$$
\lim _{\varepsilon \rightarrow 0}\left(a_{\varepsilon}\right)^{2} \sigma_{\varepsilon}=\mathbb{R}^{+}
$$

Theorem 1.6 is consistent with Weyl's asymptotic distribution of eigenvalues for the Laplacian. Indeed, if there were no periodic heterogeneities (i.e. if the coefficients $a_{k \ell}(y)$ were constant), then Weyl's result would imply that the renormalized limit of the spectrum is always the entire positive half line. The reader can find a proof of Theorem 1.6 in the forthcoming paper [3].

With Theorem 1.5 we still have the important question of how to characterize explicitly the boundary layer spectrum. Indeed, this definition of $\sigma_{\text {boundary }}$ is not very enlightening, because it does not characterize this part of the limit of $\epsilon^{2} \sigma_{\epsilon}$ as the spectrum of an operator associated with the boundary $\partial \Omega$ of $\Omega$. In particular, it is not clear whether $\sigma_{\text {boundary }}$ is empty or included in $\sigma_{\text {Bloch }}$. There is a subtle point here : the answer depends on the choice 
of the sequence $\epsilon$. A striking result has recently been obtained by C. Castro and E. Zuazua [11] when the sequence $\epsilon$ takes all real values close to 0 .

Theorem 1.7. Let $\varepsilon$ be the sequence of all real numbers in the interval $\left(0, \varepsilon_{0}\right)$ with $\varepsilon_{0}>0$. Then

$$
\lim _{\varepsilon \rightarrow 0} \varepsilon^{2} \sigma_{\varepsilon}=\mathbb{R}^{+},
$$

which means that the boundary layer spectrum $\sigma_{\text {boundary }}$ must necessarily fill the gaps of the Bloch spectrum $\sigma_{\text {Bloch }}$.

In Theorem 1.7. it is crucial that the sequence $\varepsilon$ takes all possible values near 0 . For a general domain $\Omega$ and a general sequence of periods $\varepsilon$, we do not know how to characterize $\sigma_{\text {boundary. }}$. On the contrary, if $\varepsilon$ is a discrete sequence and $\Omega$ has a piecewise flat boundary, we obtain a complete characterization of $\sigma_{\text {boundary }}$ which may not any longer fill the gaps of $\sigma_{\text {Bloch }}$. The reader is referred to [3], where is considered the case where $\Omega$ is a rectangle with integer dimensions and the sequence $\varepsilon$ is given exactly by $\varepsilon_{n}=1 / n, n \in \mathbb{N}^{*}$, which means that $\Omega$ is always a union of entire periodic cells of size $\varepsilon_{n}$. Suitable generalizations of the above methods led in this case to a complete characterization of $\sigma_{\text {boundary }}$. In particular, we generalize the two-scale convergence for treating the case of boundary layers.

Concluding this Introduction, let us mention that the Bloch wave approach has already been applied to other spectral problems. The interested reader can find a systematic presentation of the method as well as several applications in [12]; see also [4], [5], [13], [15].

\section{Classical Homogenization Results}

Our plan to prove Theorem 1.2 is as follows: in $\S 2.1$, we introduce Bloch waves at $\varepsilon$-scale and Bloch transforms and we analyze their behaviour as $\varepsilon \rightarrow 0$. Though it is not strictly rigorous, it will be instructive to begin by considering the special case where $\Omega=\mathbb{R}^{N}$. The differential equation $\mathcal{A}^{\varepsilon} u^{\varepsilon}=f$ in $\mathbb{R}^{N}$ can be easily transformed to a set of algebraic equations for the Bloch transforms (see equation (17)). We show next that the energy of $u^{\varepsilon}$ contained in all Bloch modes except the first one goes to zero (Proposition 2.2). Our next aim is to pass to the limit in the equation (17) corresponding to the first Bloch mode. We prove that the first Bloch transform tends to the usual Fourier transform (Proposition 2.3). Thus, we see that the Bloch waves representing periodic medium tend to Fourier waves representing the homogenized medium.

As will be evident from the analysis below, the passage to the limit in equation (17) requires smoothness of the first Bloch mode and also of the first Bloch eigenvalue when $\eta$ is in a neighbourhood of the origin. These results have been established in [14], §2, so we will assume that for small values of $\eta$ one can choose $\phi_{1}(y ; \eta)$ in a smooth way, and that this choice is done in such a way that

$$
\phi_{1}(y ; 0) \equiv|Y|^{-\frac{1}{2}}
$$

Note that the constant $|Y|^{-\frac{1}{2}}$ is dictated by the normalization condition that the norm in $L_{\#}^{2}(Y)$ is unity.

A natural question which arises in the limiting-passage step is to know what happens to periodic oscillations if we work in the Fourier space. Without going into details let us see this heuristically. Denote by $\xi$ and $\eta$ the variables dual to $x$ and $y$ in the Fourier sense. Since the Fourier transform of a function depending on $x / \varepsilon$ is a function of $\varepsilon \xi$, we have the relation $\eta=\varepsilon \xi$. Thus, if we replace each derivative $\partial / \partial x_{j}$, as is usual in Fourier analysis, by $\xi_{j}$ which is equal to $\varepsilon^{-1} \eta_{j}$, we see that we accumulate negative powers of $\varepsilon$. In order to overcome this, we use Taylor expansion of $\lambda_{1}(\eta)$. We know already that $\lambda_{1}(0)=0$. To compensate the negative powers of $\varepsilon$ and have a finite limit as $\varepsilon \rightarrow 0$, we need to show that $\lambda_{1}^{\prime}(0)$ also vanishes. We do this in Proposition 2.4. Once done, this shows (not rigorously 
though) that the homogenized matrix obtained in this method is nothing but $\frac{1}{2} \lambda_{1}^{\prime \prime}(0)$, i.e., $\frac{1}{2}$ times the Hessien matrix of $\lambda_{1}$ at the origin, which is already shown to be a critical point for $\lambda_{1}$. Another purpose of Proposition 2.4 is to calculate the Hessien of $\lambda_{1}$ and identify the homogenized matrix obtained in this method with the one obtained via classical means in [8].

In order to make the passage to the limit in (17) more rigorous, we must localize the equation $\mathcal{A}^{\varepsilon} u^{\varepsilon}=f$ in $\Omega$ by means of a cut-off function, as demanded by Proposition 2.3. This reduces the problem in $\Omega$ to another one in $\mathbb{R}^{N}$ for which our foregoing arguments apply. The details are presented in $\S 2.3$.

\subsection{Preliminaries for the proof of Theorem $\mathbf{1 . 2}$}

The first step is to consider the case $\Omega=\mathbb{R}^{N}$ and express the equation $\mathcal{A}^{\varepsilon} u^{\varepsilon}=f$ in $\mathbb{R}^{N}$ in an equivalent way in terms of the Bloch coefficients of $u^{\varepsilon}$ and $f$. In order to do this, we introduce Bloch eigenvalues $\left\{\lambda_{m}^{\varepsilon}(\xi)\right\}_{m=1}^{\infty}$ and eigenvectors $\left\{\phi_{m}^{\varepsilon}(x ; \xi)\right\}_{m=1}^{\infty}$ in the $\varepsilon$-scale. By homothecy we have:

$$
\lambda_{m}^{\varepsilon}(\xi)=\varepsilon^{-2} \lambda_{m}(\eta), \quad \phi_{m}^{\varepsilon}(x ; \xi)=\phi_{m}(y ; \eta),
$$

where $\lambda_{m}(\eta), \phi_{m}(y ; \eta)$ were already introduced in $\S 1.1$ and $(x, \xi)$ and $(y, \eta)$ are related by

$$
y=\frac{x}{\varepsilon}, \quad \eta=\varepsilon \xi
$$

Recall that $y \in Y=\left[0,2 \pi\left[{ }^{N}\right.\right.$ and $\left.\eta \in Y^{\prime}=\right]-\frac{1}{2}, \frac{1}{2}\left[{ }^{N}\right.$. Hence $\xi \in \varepsilon^{-1} Y^{\prime}=\left[-\frac{\varepsilon^{-1}}{2}, \frac{\varepsilon^{-1}}{2}\left[{ }^{N}\right.\right.$. The following fundamental result regarding Bloch waves is proved in [12]:

Theorem 2.1. Let $g \in L^{2}\left(\mathbb{R}_{x}^{N}\right), m \in \mathbb{N}$ and $\xi \in \varepsilon^{-1} Y^{\prime}$ be given. The $m^{\text {th }}$ Bloch coefficient of $g$ (at the $\varepsilon$-scale) is defined as follows:

$$
\hat{g}_{m}^{\varepsilon}(\xi)=\varepsilon^{-\frac{N}{2}} \int_{\mathbb{R}^{N}} g(x) e^{-i \xi \cdot x} \bar{\phi}_{m}^{\varepsilon}(x ; \xi) d x .
$$

Then the following inverse formula holds:

$$
g(x)=\varepsilon^{\frac{N}{2}} \int_{\varepsilon^{-1} Y^{\prime}} \sum_{m=1}^{\infty} \hat{g}_{m}^{\varepsilon}(\xi) e^{i \xi x} \phi_{m}^{\varepsilon}(x ; \xi) d \xi .
$$

Further, we have Parseval's Identity:

$$
\varepsilon^{-N} \int_{\mathbb{R}^{N}}|g(x)|^{2} d x=\int_{\varepsilon^{-1} Y^{\prime}} \sum_{m=1}^{\infty}\left|\hat{g}_{m}^{\varepsilon}(\xi)\right|^{2} d \xi .
$$

More generally, Plancherel Identity holds:

$$
\varepsilon^{-N} \int_{\mathbb{R}^{N}} g(x) \bar{h}(x) d x=\int_{\varepsilon^{-1} Y^{\prime}} \sum_{m=1}^{\infty} \hat{g}_{m}^{\varepsilon}(\xi) \overline{\hat{h}_{m}^{\varepsilon}}(\xi) d \xi \quad \forall g, h \in L^{2}\left(\mathbb{R}_{x}^{N}\right) .
$$

Thanks to the above result and the relation

$$
\mathcal{A}^{\varepsilon}\left(e^{i \xi \cdot x} \phi_{m}^{\varepsilon}(x ; \xi)\right)=\lambda_{m}^{\varepsilon}(\xi) e^{i \xi \cdot x} \phi_{m}^{\varepsilon}(x ; \xi),
$$

we see that the equation $\mathcal{A}^{\varepsilon} u^{\varepsilon}=f$ in $\mathbb{R}^{N}$ is equivalent to

$$
\hat{f}_{m}^{\varepsilon}(\xi)=\lambda_{m}^{\varepsilon}(\xi) \hat{u}_{m}^{\varepsilon}(\xi) \quad \forall m \geq 1, \forall \xi \in \varepsilon^{-1} Y^{\prime}
$$


Our goal is to pass to the limit in this system. Our first claim is that one can neglect all the equations corresponding to $m \geq 2$.

Proposition 2.2. Let

$$
v^{\varepsilon}(x)=\varepsilon^{\frac{N}{2}} \int_{\varepsilon^{-1} Y^{\prime}} \sum_{m=2}^{\infty} \hat{u}_{m}^{\varepsilon}(\xi) e^{i \xi \cdot x} \phi_{m}^{\varepsilon}(x ; \xi) d \xi .
$$

Then $\left\|v^{\varepsilon}\right\|_{L^{2}\left(\mathbb{R}^{N}\right)} \leq c \varepsilon$.

Proof. We have

$$
\int_{\mathbb{R}^{N}} \mathcal{A}^{\varepsilon} u^{\varepsilon} \bar{u}^{\varepsilon}=\int_{\mathbb{R}^{N}} f \bar{u}^{\varepsilon} .
$$

By Plancherel Identity, we deduce that

$$
\begin{aligned}
\beta \int_{\mathbb{R}^{N}}\left|\nabla u^{\varepsilon}\right|^{2} & \geq \varepsilon^{N} \int_{\varepsilon^{-1} Y^{\prime}} \sum_{m=1}^{\infty} \hat{f}_{m}^{\varepsilon}(\xi) \overline{\hat{u}_{m}^{\varepsilon}(\xi)} d \xi \\
& =\varepsilon^{N} \int_{\varepsilon^{-1} Y^{\prime}} \sum_{m=1}^{\infty} \lambda_{m}^{\varepsilon}(\xi)\left|\hat{u}_{m}^{\varepsilon}(\xi)\right|^{2} d \xi .
\end{aligned}
$$

where the constant $\beta$ is equal to $\max _{k, \ell}\left\|a_{k, \ell}\right\|_{L^{\infty}(Y)}$. As a simple consequence of the min-max principle, it can be proved that

$$
\lambda_{m}(\eta) \geq \lambda_{2}(\eta) \geq \lambda_{2}^{(N)}>0 \quad \forall m \geq 2, \quad \forall \eta \in Y^{\prime},
$$

where $\lambda_{2}^{(N)}$ is the second eigenvalue of the eigenvalue problem for $\mathcal{A}$ in the cell $Y$ with Neumann boundary condition on $\partial Y$. Then

$$
\varepsilon^{N} \int_{\varepsilon^{-1} Y^{\prime}} \sum_{m=2}^{\infty}\left|\hat{u}_{m}^{\varepsilon}(\xi)\right|^{2} d \xi \leq c \varepsilon^{2} .
$$

By Parseval's Identity, the left side is equal to $\left\|v^{\varepsilon}\right\|_{L^{2}\left(\mathbb{R}^{N}\right)}^{2}$. This finishes the proof.

With the aim of passing to the limit in (17) with $m=1$, we now prove

Proposition 2.3. Let $\left\{g^{\varepsilon}\right\}$ be a sequence in $L^{2}\left(\mathbb{R}^{N}\right)$ and $g$ be an element of $L^{2}\left(\mathbb{R}^{N}\right)$. Assume that there is a fixed compact set $K$ such that $\operatorname{supp} g^{\varepsilon} \subseteq K, \forall \varepsilon$. Thus, if $g^{\varepsilon} \rightarrow g$ in $L^{2}\left(\mathbb{R}^{N}\right)$-weakly then $\varepsilon^{N / 2} \hat{g}_{1}^{\varepsilon} \rightarrow \hat{g}$ in $L_{\text {loc }}^{2}\left(\mathbb{R}^{N}\right)$-weakly, where $\hat{g}_{1}^{\varepsilon}$ denotes the first Bloch transform of $g^{\varepsilon}$ and $\hat{g}$ is the usual Fourier-Plancherel transform of $g$.

Proof. It is understood that $\hat{g}_{1}^{\varepsilon}(\xi)$ which is apriori defined for $\left.\xi \in \varepsilon^{-1} Y^{\prime}=\right]-\frac{\varepsilon^{-1}}{2}, \frac{\varepsilon^{-1}}{2}\left[{ }^{N}\right.$ is extended by zero outside $\varepsilon^{-1} Y^{\prime}$. We write

$$
\varepsilon^{N / 2} \hat{g}_{1}^{\varepsilon}(\xi)=\int_{\mathbb{R}^{N}} g^{\varepsilon}(x) e^{-i x \cdot \xi} \bar{\phi}_{1}\left(\frac{x}{\varepsilon} ; 0\right) d x+\int_{K} g^{\varepsilon}(x) e^{-i x \cdot \xi}\left(\bar{\phi}_{1}\left(\frac{x}{\varepsilon} ; \varepsilon \xi\right)-\bar{\phi}_{1}\left(\frac{x}{\varepsilon} ; 0\right)\right) d x .
$$

Since $\phi_{1}(y ; 0)=|Y|^{-1 / 2}=(2 \pi)^{-N / 2}$, we see that the first term is nothing but the Fourier transform of $g^{\varepsilon}$ and so it converges weakly to $g(\xi)$ in $L^{2}\left(\mathbb{R}^{N}\right)$. The second term is bounded by

$$
\left\|g^{\varepsilon}\right\|_{L^{2}\left(\mathbb{R}^{N}\right)}\left[\int_{K}\left|\phi_{1}\left(\frac{x}{\varepsilon} ; \varepsilon \xi\right)-\phi_{1}\left(\frac{x}{\varepsilon} ; 0\right)\right|^{2} d x\right]^{1 / 2} \leq c\left\|\phi_{1}(y ; \varepsilon \xi)-\phi_{1}(y ; 0)\right\|_{L^{2}(Y)} .
$$

Here is where some regularity of the first Bloch mode $\eta \rightarrow \phi_{1}(\cdot, \eta) \in L_{\#}^{2}(Y)$ is required when $\eta$ is near 0 . Analyticity of this map is established in [14], but here we simply use the fact 
that the above map is Lipschitz. Thus we conclude that the second term in the right side of (18) is bounded above by $c \varepsilon \xi$. Thus if $|\xi| \leq M$, we see that it is bounded above by $c M \varepsilon$ and so, in particular, it converges to zero in $L_{l o c}^{\infty}\left(\mathbb{R}^{N}\right)$, completing the proof of Proposition 2.3.

\subsection{Identification of the homogenized coefficients}

The aim of this paragraph is to give a different expression for the homogenized matrix $\left(q_{k \ell}\right)$ in terms of the first Bloch eigenvalue $\lambda_{1}(\eta)$. Let us recall the classical expression for $\left(q_{k \ell}\right)$ from $[8]$ :

$$
q_{k \ell}=\frac{1}{|Y|} \int_{Y} a_{k \ell} d y+\frac{1}{|Y|} \int_{Y} a_{k m} \frac{\partial \chi_{\ell}}{\partial y_{m}} d y \quad \forall k, \ell=1, \ldots, N .
$$

where $\chi_{k}$ is the unique solution (defined up to an additive constant) of the following problem with periodic boundary conditions:

$$
\mathcal{A} \chi_{k}=\frac{\partial a_{k \ell}}{\partial y_{\ell}} \quad \text { in } \quad \mathbb{R}^{N}, \quad \chi_{k} \quad Y \text {-periodic, } \forall k=1, \ldots, N .
$$

We then have

Proposition 2.4. The origin is a critical point of the first Bloch eigenvalue:

$$
\frac{\partial \lambda_{1}}{\partial \eta_{k}}(0)=0 \quad \forall k=1, \ldots, N .
$$

Further, the Hessien of $\lambda_{1}$ at $\eta=0$ is given by

$$
\frac{1}{2} \frac{\partial^{2} \lambda_{1}}{\partial \eta_{k} \partial \eta_{\ell}}(0)=q_{k \ell} \quad \forall k, \ell=1, \ldots, N .
$$

The derivatives of the first Bloch mode can also be calculated and they are as follows:

$$
\frac{\partial \phi_{1}}{\partial \eta_{k}}(y ; 0)=i|Y|^{-\frac{1}{2}} \chi_{k}(y) \quad \forall k=1, \ldots, N .
$$

Proof. Given the fact that $\eta \rightarrow \lambda_{1}(\eta)$ and $\eta \rightarrow \phi_{1}(y ; \eta)$ are smooth, it is straightforward to compute their derivatives at $\eta=0$. Indeed, it is enough to differentiate the eigenvalue equation $\mathcal{A}(\eta) \phi_{1}(\cdot ; \eta)=\lambda_{1}(\eta) \phi_{1}(\cdot ; \eta)$ with respect to $\eta$ twice and evaluate at $\eta=0$. Since the computations are classical, we only present the essential steps. We obtain

$$
\begin{gathered}
\frac{\partial \lambda_{1}}{\partial \eta_{k}}(\eta)=\left(\frac{\partial \mathcal{A}(\eta)}{\partial \eta_{k}} \phi_{1}(\cdot ; \eta), \phi_{1}(\cdot ; \eta)\right), \\
\left(\mathcal{A}(\eta)-\lambda_{1}(\eta)\right) \frac{\partial \phi_{1}}{\partial \eta_{k}}(\cdot ; \eta)+\left[\frac{\partial \mathcal{A}(\eta)}{\partial \eta_{k}}-\frac{\partial \lambda_{1}}{\partial \eta_{k}}(\eta)\right] \phi_{1}(\cdot ; \eta)=0, \\
\frac{1}{2} \frac{\partial^{2} \lambda_{1}}{\partial \eta_{k} \partial \eta_{\ell}}(\eta)=\left(a_{k \ell} \phi_{1}(\cdot ; \eta), \phi_{1}(\cdot ; \eta)\right) \\
+\frac{1}{2}\left(\left[\frac{\partial \mathcal{A}(\eta)}{\partial \eta_{k}}-\frac{\partial \lambda_{1}}{\partial \eta_{k}}(\eta)\right] \frac{\partial \phi_{1}}{\partial \eta_{\ell}}(\cdot ; \eta), \phi_{1}(\cdot ; \eta)\right) \\
+\frac{1}{2}\left(\left[\frac{\partial \mathcal{A}(\eta)}{\partial \eta_{\ell}}-\frac{\partial \lambda_{1}}{\partial \eta_{\ell}}(\eta)\right] \frac{\partial \phi_{1}}{\partial \eta_{k}}(\cdot ; \eta), \phi_{1}(\cdot ; \eta)\right) .
\end{gathered}
$$


We know already that $\lambda_{1}(0)=0$ and by our choice $\phi_{1}(y ; 0)=|Y|^{-\frac{1}{2}}$. If we use this information in the above relations and evaluate them at $\eta=0$, we get successively (21), (23) and (22).

Before taking up the rigorous proof of Theorem 1.2, we pass to the limit in relation (17) in a heuristic manner to see the homogenized equation obtained via Bloch-wave method. Let us take $m=1$ in (17) and multiply both sides by $\varepsilon^{N / 2}$ :

$$
\varepsilon^{-2} \lambda_{1}(\varepsilon \xi) \varepsilon^{N / 2} \hat{u}_{1}^{\varepsilon}(\xi)=\varepsilon^{N / 2} \hat{f}_{1}^{\varepsilon}(\xi)
$$

Expanding $\lambda_{1}(\varepsilon \xi)$ by Taylor's Formula around $\xi=0$, and using Proposition 2.4, we get

$$
\left[\frac{1}{2} \frac{\partial^{2} \lambda_{1}}{\partial \eta_{k} \partial \eta_{\ell}}(0) \xi_{k} \xi_{\ell}+O\left(\varepsilon \xi^{3}\right)\right] \varepsilon^{N / 2} \hat{u}_{1}^{\varepsilon}(\xi)=\varepsilon^{N / 2} \hat{f}_{1}^{\varepsilon}(\xi)
$$

A simple passage to the limit yields

$$
\frac{1}{2} \frac{\partial^{2} \lambda_{1}}{\partial \eta_{k} \partial \eta_{\ell}}(0) \xi_{k} \xi_{\ell} \hat{u}^{*}(\xi)=\hat{f}(\xi)
$$

where, we recall, $u^{*}$ is the $L^{2}$-weak limit of $u^{\varepsilon}$.

Thanks to (22), the above equation is nothing but the homogenized equation in the Fourier space, i.e., it is just the Fourier transform of the usual homogenized equation. It can be noted that the passage to the limit here is more direct than in Tartar's method because no derivatives are involved in (24). However, there is one flaw in our argument of letting $\varepsilon \rightarrow 0$ in (24). Strictly speaking, we cannot apply Proposition 2.3 since $u^{\varepsilon}$ need not have uniform compact support. A natural way to overcome this difficulty is to use the cut-off function technique to localize the equation. This is what we carry out in the next paragraph.

\subsection{Proof of Theorem $\mathbf{1 . 2}$}

Let $\phi \in \mathcal{D}(\Omega)$ be arbitrary. If $u^{\varepsilon}$ satisfies $\mathcal{A}^{\varepsilon} u^{\varepsilon}=f$ in $\Omega$ then its localization $\phi u^{\varepsilon}$ satisfies

$$
\mathcal{A}^{\varepsilon}\left(\phi u^{\varepsilon}\right)=\phi f+g^{\varepsilon}+h^{\varepsilon} \quad \text { in } \mathbb{R}^{N},
$$

where

$$
\begin{aligned}
g^{\varepsilon} & =-2 a_{k \ell}^{\varepsilon} \frac{\partial u^{\varepsilon}}{\partial x_{\ell}} \frac{\partial \phi}{\partial x_{k}}-a_{k \ell}^{\varepsilon} \frac{\partial^{2} \phi}{\partial x_{k} \partial x_{\ell}} u^{\varepsilon}=-2 \sigma_{k}^{\varepsilon} \frac{\partial \phi}{\partial x_{k}}-a_{k \ell}^{\varepsilon} \frac{\partial^{2} \phi}{\partial x_{k} \partial x_{\ell}} u^{\varepsilon}, \\
h^{\varepsilon} & =-\frac{\partial a_{k \ell}^{\varepsilon}}{\partial x_{k}} \frac{\partial \phi}{\partial x_{\ell}} u^{\varepsilon} .
\end{aligned}
$$

Using the arguments outlined above leading to (26), we can pass to the limit in (27): since $\phi u^{\varepsilon}$ is bounded in $H^{1}\left(\mathbb{R}^{N}\right)$, we can neglect all the harmonics corresponding to $m \geq 2$. The component corresponding to $m=1$ yields at the limit

$$
\frac{1}{2} \frac{\partial^{2} \lambda_{1}}{\partial \eta_{k} \partial \eta_{\ell}}(0) \xi_{k} \xi_{\ell}\left(\widehat{\phi u^{*}}\right)(\xi)=\widehat{(\phi f)}(\xi)+\lim _{\varepsilon \rightarrow 0} \varepsilon^{\frac{N}{2}} \hat{g}_{1}^{\varepsilon}(\xi)+\lim _{\varepsilon \rightarrow 0} \varepsilon^{\frac{N}{2}} \hat{h}_{1}^{\varepsilon}(\xi),
$$

where $\hat{g}_{1}^{\varepsilon}, \hat{h}_{1}^{\varepsilon}$ are the first Bloch transform of $g^{\varepsilon}$ and $h^{\varepsilon}$ respectively. The sequence $\left\{\sigma_{k}^{\varepsilon}\right\}$ is bounded in $L^{2}(\Omega)$, we can therefore extract a subsequence (still denoted by $\varepsilon$ ) which is weakly convergent in $L^{2}(\Omega)$. Let $\sigma_{k}^{*}$ denote its limit as well as its extension by zero outside $\Omega$. Using this convergence and the definition of $g^{\varepsilon}$ we see that

$$
g^{\varepsilon} \rightarrow g^{*} \stackrel{\text { def }}{=}-2 \sigma_{k}^{*} \frac{\partial \phi}{\partial x_{k}}-\mathcal{M}\left(a_{k \ell}\right) \frac{\partial^{2} \phi}{\partial x_{k} \partial x_{\ell}} u^{*} \quad \text { in } \quad L^{2}\left(\mathbb{R}^{N}\right) \text { weakly, }
$$

ESAIM: PROC., VOL. 3, 1998, 65-84 
where $\mathcal{M}\left(a_{k \ell}\right)$ is the average of $a_{k \ell}$ on $Y$. From Proposition 2.3, it follows that

$$
\varepsilon^{\frac{N}{2}} \hat{g}_{1}^{\varepsilon}(\xi) \rightarrow \hat{g}^{*}(\xi) \quad \text { in } \quad L_{l o c}^{2}\left(\mathbb{R}_{\xi}^{N}\right) \text { weakly }
$$

Concerning the sequence $\left\{\hat{h}_{1}^{\varepsilon}\right\}$, we cannot apply Proposition 2.3 directly because $h^{\varepsilon}$ is not bounded in $L^{2}\left(\mathbb{R}^{N}\right)$. However, following the idea of Proposition 2.3, we decompose

$$
\varepsilon^{\frac{N}{2}} \hat{h}_{1}^{\varepsilon}(\xi)=\int_{\mathbb{R}^{N}} h^{\varepsilon}(x) e^{-i x \cdot \xi} \bar{\phi}_{1}\left(\frac{x}{\varepsilon}, 0\right) d x+\int_{\mathbb{R}^{N}} h^{\varepsilon}(x) e^{-i x \cdot \xi}\left(\bar{\phi}_{1}\left(\frac{x}{\varepsilon} ; \varepsilon \xi\right)-\bar{\phi}_{1}\left(\frac{x}{\varepsilon} ; 0\right)\right) d x .
$$

The proof of Proposition 2.3 shows that the second term in the right hand of (29) tends to zero if the sequence is bounded in $L^{2}\left(\mathbb{R}^{N}\right)$. In fact, using the Taylor expansion of $\phi_{1}(y ; \eta)$ at $\eta=0$, we see that the second term is equal to

$$
-\varepsilon^{-1} \int_{\mathbb{R}^{N}} \frac{\partial a_{k \ell}}{\partial y_{k}}\left(\frac{x}{\varepsilon}\right) \frac{\partial \phi}{\partial x_{\ell}}(x) u^{\varepsilon}(x) e^{-i x \cdot \xi}\left[\varepsilon \frac{\partial \bar{\phi}_{1}}{\partial \eta_{j}}\left(\frac{x}{\varepsilon} ; 0\right) \xi_{j}+O\left(\varepsilon^{2} \xi^{2}\right)\right] d x
$$

which evidently converges in $L_{l o c}^{\infty}\left(\mathbb{R}_{\xi}^{N}\right)$ strongly to

$$
-\mathcal{M}\left(\frac{\partial a_{k \ell}}{\partial y_{k}} \frac{\partial \bar{\phi}_{1}}{\partial \eta_{j}}(y ; 0)\right) \xi_{j} \int_{\mathbb{R}^{N}} \frac{\partial \phi}{\partial x_{\ell}} u^{*} e^{-i x \cdot \xi} d x
$$

On the other hand, the first term of the right side of (29), after an integration by parts, becomes

$$
\int_{\mathbb{R}^{N}} a_{k \ell}^{\varepsilon}\left[\frac{\partial^{2} \phi}{\partial x_{k} \partial x_{\ell}} u^{\varepsilon}+\frac{\partial \phi}{\partial x_{\ell}} \frac{\partial u^{\varepsilon}}{\partial x_{k}}-i \xi_{k} \frac{\partial \phi}{\partial x_{\ell}} u^{\varepsilon}\right] e^{-i x \cdot \xi} \bar{\phi}_{1}\left(\frac{x}{\varepsilon} ; 0\right) d x .
$$

Since $\phi_{1}(y ; 0)=|Y|^{-\frac{1}{2}}$, it is easily seen that the above integral converges weakly in $L^{2}\left(\mathbb{R}^{N}\right)$ to

$$
|Y|^{-\frac{1}{2}}\left[\mathcal{M}\left(a_{k \ell}\right) \int_{\mathbb{R}^{N}} \frac{\partial^{2} \phi}{\partial x_{k} \partial x_{\ell}} u^{*} e^{-i x \cdot \xi} d x+\int_{\mathbb{R}^{N}} \sigma_{\ell}^{*} \frac{\partial \phi}{\partial x_{\ell}} e^{-i x \cdot \xi} d x-i \xi_{k} \mathcal{M}\left(a_{k \ell}\right) \int_{\mathbb{R}^{N}} \frac{\partial \phi}{\partial x_{\ell}} u^{*} e^{-i x \cdot \xi} d x\right]
$$

Using (28) and Proposition 2.4, we arrive at

$$
\left.q_{k \ell} \xi_{k} \xi_{\ell}\left(\widehat{\phi u^{*}}\right)(\xi)=\widehat{(\phi f}\right)(\xi)-|Y|^{-\frac{1}{2}} \int_{\mathbb{R}^{N}} \sigma_{k}^{*} \frac{\partial \phi}{\partial x_{k}} e^{-i x \cdot \xi} d x-i \xi_{k}|Y|^{-\frac{1}{2}} q_{k \ell} \int_{\mathbb{R}^{N}} \frac{\partial \phi}{\partial x_{\ell}} u^{*} e^{-i x \cdot \xi} d x
$$

This can be rewritten as

$$
\left[\mathcal{A}^{*}\left(\widehat{\phi} u^{*}\right)\right](\xi)=\widehat{(\phi f)}(\xi)-|Y|^{-\frac{1}{2}} \int_{\mathbb{R}^{N}} \sigma_{k}^{*} \frac{\partial \phi}{\partial x_{k}} e^{-i x \cdot \xi} d x-i \xi_{k}|Y|^{-\frac{1}{2}} q_{k \ell} \int_{\mathbb{R}^{N}} \frac{\partial \phi}{\partial x_{\ell}} u^{*} e^{-i x \cdot \xi} d x .
$$

We can call this localized homogenized equation in the Fourier space. The conclusions of Theorem 1.2 are easy consequences of this equation. In fact, taking inverse Fourier transform of (30) we obtain

$$
\mathcal{A}^{*}\left(\phi u^{*}\right)=\phi f-\sigma_{k}^{*} \frac{\partial \phi}{\partial x_{k}}-q_{k \ell} \frac{\partial}{\partial x_{k}}\left(\frac{\partial \phi}{\partial x_{\ell}} u^{*}\right) \text { in } \mathbb{R}^{N} .
$$

On the other hand, we can calculate $\mathcal{A}^{*}\left(\phi u^{*}\right)$ directly:

$$
\mathcal{A}^{*}\left(\phi u^{*}\right)=-q_{k \ell} \frac{\partial^{2} \phi}{\partial x_{k} \partial x_{\ell}} u^{*}-2 q_{k \ell} \frac{\partial \phi}{\partial x_{k}} \frac{\partial u^{*}}{\partial x_{\ell}}+\phi \mathcal{A}^{*} u^{*} \text { in } \mathbb{R}^{N} .
$$


A simple comparison between (31) and (32) yields

$$
\phi\left(\mathcal{A}^{*} u^{*}-f\right)=\left(q_{k \ell} \frac{\partial u^{*}}{\partial x_{\ell}}-\sigma_{k}^{*}\right) \frac{\partial \phi}{\partial x_{k}} \quad \text { in } \mathbb{R}^{N} .
$$

Since the above relation is true for all $\phi$ in $\mathcal{D}(\Omega)$, the desired conclusions follow. .

\section{Asymptotics of the wave equation spectrum}

This section is devoted to the proof of Theorem 1.5. For a better organization of the exposition, we shall divide the proof into two main steps.

\subsection{Bloch wave homogenization}

Studying a specific spectral problem which arises in fluid-solid interactions, G. Allaire and C. Conca recently introduced in [5] a new method of homogenization, the so-called Blochwave homogenization method (which is a merge of two-scale convergence and Bloch-wave decomposition), which has been very useful for tacking the asymptotic behaviour of the spectrum of periodic structures. By means of this Bloch-wave homogenization, we prove in this paragraph that

$$
\sigma_{\text {Bloch }} \subset \sigma^{\infty} \text {. }
$$

where $\sigma^{\infty}$ is used as a shorthand of $\lim _{\varepsilon \rightarrow 0} \varepsilon^{2} \sigma^{\varepsilon}$. To this end, let us rewrite the spectral problem (10) as follows: Find $\left(\mu^{\varepsilon}, v^{\varepsilon}\right), v^{\varepsilon} \not \equiv 0$, such that

$$
\varepsilon^{2} \mathcal{A}^{\varepsilon} v^{\varepsilon}+v^{\varepsilon}=\frac{1}{\mu^{\varepsilon}} v^{\varepsilon} \quad \text { in } \quad \Omega, \quad v^{\varepsilon} \in H_{0}^{1}(\Omega) .
$$

Observe that passing from (10) to (34) leaves the eigenfunctions invariant and changes the eigenvalues $\lambda_{m}^{\varepsilon}$ into $\mu_{m}^{\varepsilon}=1 /\left(1+\varepsilon^{2} \lambda_{m}^{\varepsilon}\right)$ and hence, $\mu^{\varepsilon} \sim 1$ iff $\lambda^{\varepsilon} \sim \varepsilon^{-2}$.

To resolve (34), it is a classical technique to introduce the so-called Green's operator $S^{\varepsilon} \in \mathcal{L}\left(L^{2}(\Omega)\right)$ which is defined as $S^{\varepsilon} f=u^{\varepsilon}$, where $u^{\varepsilon}$ is the unique solution of

$$
\varepsilon^{2} \mathcal{A}^{\varepsilon} u^{\varepsilon}+u^{\varepsilon}=f \quad \text { in } \Omega, \quad u^{\varepsilon} \in H_{0}^{1}(\Omega) .
$$

The starting point in order to characterize $\sigma^{\infty}$ is to pass to the limit in (35). Well now, it is an easy exercise in perturbation's theory to show that $u^{\varepsilon}$ converges weakly in $L^{2}(\Omega)$ to $f$. This implies that $S^{\varepsilon}$ converges weakly to the identity operator, which is a useless result concerning its spectrum. This appeals to obtain a strong convergence for $S^{\varepsilon}$ and the main idea in obtaining this is to extend the operator $S^{\varepsilon}$ by embedding $L^{2}(\Omega)$ into a larger space of two-scale functions. Of course, the extension of $S^{\varepsilon}$ must be done in such a way as to essentially maintain the same spectrum as $S^{\varepsilon}$. With this in mind, for any positive integer $K \geq 1$, denoting by $K Y$ the cube $[0, K]^{N}$, we define an extension operator $S_{K}^{\varepsilon} \in \mathcal{L}\left(L^{2}\left(\Omega ; L_{\#}^{2}(K Y)\right)\right)$ by

$$
S_{K}^{\varepsilon}=E_{K}^{\varepsilon} S^{\varepsilon} P_{K}^{\varepsilon},
$$

where $P_{K}^{\varepsilon}$ and $E_{K}^{\varepsilon}$ are respectively a projection from $L^{2}\left(\Omega ; L_{\#}^{2}(K Y)\right)$ into $L^{2}(\Omega)$ and an extension from $L^{2}(\Omega)$ into $L^{2}\left(\Omega ; L_{\#}^{2}(K Y)\right)$. To insure that $S_{K}^{\varepsilon}$ is still self-adjoint, we ask $P_{K}^{\varepsilon}$ and $E_{K}^{\varepsilon}$ to be adjoint one from the other. To insure that $S^{\varepsilon}$ and $S_{K}^{\varepsilon}$ have the same spectrum, denoted by $\tilde{\sigma}^{\varepsilon}$, we ask the product $P_{K}^{\varepsilon} E_{K}^{\varepsilon}$ to be equal to the identity in $L^{2}(\Omega)$. Such conditions are satisfied by

$$
\left\{\begin{array}{l}
\forall \varphi \in L^{2}\left(\Omega ; L_{\#}^{2}(K Y)\right), \quad\left(P_{K}^{\varepsilon} \varphi\right)(x)=\sum_{i=1}^{n(\varepsilon)} \chi_{i}^{\varepsilon}(x) \frac{1}{(K \varepsilon)^{N}} \int_{Y_{i}^{\varepsilon}} \varphi\left(x^{\prime}, \frac{x}{\varepsilon}\right) d x^{\prime}, \\
\forall f \in L^{2}(\Omega), \quad\left(E_{K}^{\varepsilon} f\right)(x, y)=\sum_{i=1}^{n(\varepsilon)} \chi_{i}^{\varepsilon}(x) f\left(x_{i}^{\varepsilon}+\varepsilon y\right),
\end{array}\right.
$$

ESAIM: Proc., VOL. 3, 1998, 65-84 
where the family $\left(Y_{i}^{\varepsilon}\right)_{1 \leq i \leq n(\varepsilon)}$ of non-overlapping cells of the type $[0 ; K \varepsilon]^{N}$ covers $\Omega\left(\chi_{i}^{\varepsilon}\right.$ is the characteristic function of $Y_{i}^{\varepsilon}$ and $x_{i}^{\varepsilon}$ its origin).

Proposition 3.1. The sequence $S_{K}^{\varepsilon}$ converges strongly to a limit operator $S_{K}$ in the sense that, for any $\varphi(x, y) \in L^{2}\left(\Omega ; L_{\#}^{2}(K Y)\right), S_{K}^{\varepsilon} \varphi$ converges strongly to $S_{K} \varphi$ in $L^{2}\left(\Omega ; L_{\#}^{2}(K Y)\right)$ and $S_{K} \varphi=u_{K}$ is the unique solution in $L^{2}\left(\Omega ; H_{\#}^{1}(K Y)\right)$ of

$$
\mathcal{A} u_{K}+u_{K}=\varphi \text { in } \Omega \times K Y \text {. }
$$

Moreover, $S_{K}$ is a self-adjoint non-compact operator in $L^{2}\left(\Omega ; L_{\#}^{2}(K Y)\right)$.

The convergence of $S_{K}^{\varepsilon}$ to $S_{K}$ cannot be uniform since $S_{K}^{\varepsilon}$ is compact, but not $S_{K}$. Thus, from Proposition 2.1.11 in [5] (or Proposition I.4.10 in [12]), we deduce the lower semi-continuity of the spectrum, i.e.,

Corollary 3.2. Let $\sigma_{K}$ denote the spectrum of $S_{K}$. Since $S_{K}^{\varepsilon}$ converges strongly to $S_{K}$, we have

$$
\sigma_{K} \subset \lim _{\varepsilon \rightarrow 0} \tilde{\sigma}_{\varepsilon}
$$

The key ingredient in the proof of Proposition 3.1 is the notion of two-scale convergence introduced in [2], [26], that we briefly recall in the sequel.

Lemma 3.3. Let $u^{\varepsilon}$ be a bounded sequence in $L^{2}(\Omega)$. Then there exists a subsequence, which we still denote by $\varepsilon$, and a limit function $u^{0}(x, y) \in L^{2}(\Omega \times Y)$ such that

$$
\lim _{\varepsilon \rightarrow 0} \int_{\Omega} u^{\varepsilon}(x) \varphi\left(x, \frac{x}{\varepsilon}\right) d x=\frac{1}{|Y|} \int_{\Omega} \int_{Y} u^{0}(x, y) \varphi(x, y) d x d y
$$

for all functions $\varphi(x, y) \in L^{2}\left(\Omega ; \mathrm{C}_{\#}^{0}(Y)\right)$.

Proof of Proposition 3.1. Let $\psi^{\varepsilon}(x, y)$ be a sequence converging weakly to $\psi(x, y)$ in the space $L^{2}\left(\Omega ; L_{\#}^{2}(K Y)\right)$. For any $\varphi \in L^{2}\left(\Omega ; L_{\#}^{2}(K Y)\right)$, we need to show that

$$
\lim _{\varepsilon \rightarrow 0} \int_{\Omega} \int_{K Y}\left(S_{K}^{\varepsilon} \varphi\right) \psi^{\varepsilon} d x d y=\int_{\Omega} \int_{K Y}\left(S_{K} \varphi\right) \psi d x d y .
$$

By definition of $S_{K}^{\varepsilon}$, one has

$$
\frac{1}{K^{N}} \int_{\Omega} \int_{K Y}\left(S_{K}^{\varepsilon} \varphi\right) \psi^{\varepsilon} d x d y=\int_{\Omega}\left(S^{\varepsilon} P_{K}^{\varepsilon} \varphi\right)\left(E_{K}^{\varepsilon}\right)^{*} \psi^{\varepsilon} d x=\int_{\Omega} u^{\varepsilon}\left(P_{K}^{\varepsilon} \psi^{\varepsilon}\right) d x,
$$

where $u^{\varepsilon}$ is the solution of (35) with right hand side $f=P_{K}^{\varepsilon} \varphi$. Using Lemma 3.3 one can show that $u^{\varepsilon}$ two-scale converges "strongly" to the solution $u_{K}$ of (36), while the sequence $P_{K}^{\varepsilon} \psi^{\varepsilon}$ two-scale converges "weakly" to $\psi$. Then, passing to the limit in (37) yields

$$
\frac{1}{K^{N}} \int_{\Omega} \int_{K Y} u_{K} \psi d x d y=\frac{1}{K^{N}} \int_{\Omega} \int_{K Y}\left(S_{K} \varphi\right) \psi d x d y
$$

which concludes the proof (see [5] for a more detailed proof in a similar case).

To compute the spectrum of $\sigma_{K}$, we use a discrete Bloch-wave decomposition in $L_{\#}^{2}(K Y)$ (see [1], or Theorem 1.1 for the continuous case). This Bloch decomposition allows to diagonalize $S_{K}$.

Lemma 3.4. ([1]) For any function $\varphi(y) \in L_{\#}^{2}(K Y)$ there exists a unique family $\left\{\varphi_{j}(y)\right\} \in$ $L_{\#}^{2}(Y)^{K^{N}}$, indexed by a multi-index $j$ whose $N$ components belong to $\{0, \ldots, K-1\}$, such that

$$
\varphi(y)=\sum_{0 \leq j \leq K-1} \varphi_{j}(y) e^{i \frac{i \cdot y}{K}},
$$


and

$$
\frac{1}{K^{N}} \int_{K Y}|\varphi|^{2} d y=\sum_{0 \leq j \leq K-1} \int_{Y}\left|\varphi_{j}\right|^{2} d y
$$

This decomposition, denoted by $\mathcal{B}$, defines a unitary isometry from $L_{\#}^{2}(K Y)$ into $L_{\#}^{2}(Y)^{K^{N}}$.

From the above lemma, we easily deduce the following:

Proposition 3.5. The operator $S_{K}$ can be diagonalized as

$$
S_{K}=\mathcal{B}^{*} T_{K} \mathcal{B} \quad \text { with } \quad T_{K}=\operatorname{diag}\left[\left(T_{j / K}\right)_{0 \leq j \leq K-1}\right]
$$

where, for each Bloch frequency $\eta=j / K, T_{\eta}$ is defined in $\mathcal{L}\left(L^{2}\left(\Omega ; L_{\#}^{2}(Y)\right)\right)$ as $T_{\eta} \varphi=u^{0}$, where $u^{0}=u^{0}(x, y)$ is the unique solution in $L^{2}\left(\Omega ; H_{\#}^{1}(Y)\right)$ of

$$
\mathcal{A}(\eta) u^{0}+u^{0}=e^{i \eta \cdot y} \varphi \quad \text { in } \quad \Omega \times K Y .
$$

We recognize in the operators $T_{\eta}$ a simple transformation of the Green's operators associated with the Bloch spectral problems (3) or (5). To conclude the proof of (33), it suffices to remark that, as $K$ goes to infinity, the discrete set of the Bloch frequencies $j / K$ becomes dense in $Y^{\prime}$ (see [3] for more details).

\subsection{Completeness of the Bloch spectrum}

We now address the question of whether the Bloch spectrum is enough to completely characterize the limit set $\sigma^{\infty}$. In other words, we seek for what we call a result of completeness. Well now, it turns out in this case that the Bloch spectrum is usually not enough to describe $\sigma^{\infty}$ because there is another source of limiting spectrum which is not taken into account in our analysis. This source corresponds to sequences of eigenvectors of (10) concentrating near the boundary $\partial \Omega$ of $\Omega$. They behave as boundary layers in the sense that they converge strongly to zero inside the domain. It is therefore clear that the oscillations of these sequences of eigenvectors cannot be captured by a usual homogenization method, neither are they filtered in $\sigma_{\text {Bloch }}$ since the Bloch waves are insensitive to the boundary.

Following this line of reasoning, we prove in this paragraph that

$$
\sigma^{\infty}=\lim _{\varepsilon \rightarrow 0} \varepsilon^{2} \sigma^{\varepsilon} \subset \sigma_{\text {boundary }} \cup \sigma_{\text {Bloch }},
$$

where $\sigma_{\text {boundary }}$ is the so-called boundary layer spectrum, which is defined in $\$ 1.2$ (see (14)). To prove (39), we consider any sequence $\left(\lambda^{\varepsilon}, v^{\varepsilon}\right) \in \mathbb{R}^{+} \times H_{0}^{1}(\Omega)$, solutions of the spectral problem (10), such that (up to a subsequence) there exists a limit $\lambda$ satisfying

$$
\left\|v^{\varepsilon}\right\|_{L^{2}(\Omega)}=1 \quad \text { and } \quad \lim _{\varepsilon \rightarrow 0} \varepsilon^{2} \lambda^{\varepsilon}=\lambda .
$$

If, for any subset $\omega$ such that $\bar{\omega} \subset \Omega$,

$$
\lim _{\varepsilon \rightarrow 0}\left\|v^{\varepsilon}\right\|_{L^{2}(\omega)}=0
$$

then, by definition, the limit eigenvalue $\lambda$ belongs to the boundary layer spectrum. Therefore, to complete the proof of Theorem 1.5, it remains to prove that, if there exists a subset $\omega$ and a subsequence, still denoted by $\varepsilon$, such that

$$
\lim _{\varepsilon \rightarrow 0}\left\|v^{\varepsilon}\right\|_{L^{2}(\omega)}=c>0,
$$

then $\lambda$ belongs to the Bloch spectrum.

ESAIM: PROC., VOL. 3, 1998, 65-84 
Now, if assumption (41) is fulfilled, then there exists a smooth cut-off function $\varphi \in \mathcal{D}(\Omega)$ such that $\varphi \equiv 1$ in $\omega$, and defining a sequence

$$
u^{\varepsilon}=\frac{\varphi v^{\varepsilon}}{\left\|\varphi v^{\varepsilon}\right\|_{L^{2}(\Omega)}}
$$

it is easily seen that $u^{\varepsilon}$ is a sequence of quasi-eigenvectors in the sense that it has compact support in $\bar{\Omega},\left\|u^{\varepsilon}\right\|_{L^{2}\left(\mathbb{R}^{N}\right)}=1$, and it satisfies

$$
\mathcal{A}^{\varepsilon} u^{\varepsilon}=\lambda^{\varepsilon} u^{\varepsilon}+r^{\varepsilon} \quad \text { in } \quad \mathbb{R}^{N},
$$

where $r^{\varepsilon} \in L^{2}\left(\mathbb{R}^{N}\right)$ is a negligible remainder term satisfying

$$
\lim _{\varepsilon \rightarrow 0}\left\langle r^{\varepsilon}, w^{\varepsilon}\right\rangle_{H^{-1}, H_{0}^{1}(\Omega)}=0,
$$

for any sequence $w^{\varepsilon}$ such that $\left\|w^{\varepsilon}\right\|_{L^{2}(\Omega)}+\varepsilon\left\|\nabla w^{\varepsilon}\right\|_{L^{2}(\Omega)^{N}} \leq C$.

The equation (42) in terms of the Bloch transforms reduces to

$$
\lambda_{m}(\eta) \widehat{u_{m}^{\varepsilon}}\left(\frac{\eta}{\varepsilon}\right)=\varepsilon^{2} \lambda^{\varepsilon} \widehat{u_{m}^{\varepsilon}}\left(\frac{\eta}{\varepsilon}\right)+\varepsilon^{2} \widehat{r_{m}^{\varepsilon}} \quad \text { in } Y^{\prime}
$$

for all $m \geq 1$, where $\widehat{u_{m}^{\varepsilon}}$ is the $m^{t h}$ Bloch coefficient of $u^{\varepsilon}$. Let us multiply these equations by $\psi_{m}(\eta) \overline{\widehat{u_{m}^{\varepsilon}}(\eta / \varepsilon)}$, where $\psi_{m}(\eta)$ is a given continuous function in $\mathrm{C}_{\#}^{0}\left(\overline{Y^{\prime}}\right)$. Adding up on $m$ and integrating in $\eta$, thanks to Plancherel Identity, we obtain the following key relation

$$
\sum_{m \geq 1} \int_{Y^{\prime}} \psi_{m}(\eta)\left(\lambda_{m}(\eta)-\varepsilon^{2} \lambda^{\varepsilon}\right)\left|\widehat{u_{m}^{\varepsilon}}\left(\frac{\eta}{\varepsilon}\right)\right|^{2} d \eta=O(\varepsilon)
$$

where $O(\varepsilon)$ tends to zero with $\varepsilon$.

For each $m \geq 1$, we associate to $u^{\varepsilon}$ a function $h_{m}^{\varepsilon}$ defined for $\eta \in Y^{\prime}$ by

$$
h_{m}^{\varepsilon}(\eta)=\left|\widehat{u_{m}^{\varepsilon}}\left(\frac{\eta}{\varepsilon}\right)\right|^{2} .
$$

Since $u^{\varepsilon}$ has a unit norm in $L^{2}\left(\mathbb{R}^{N}\right)$, by Parseval's Identity we have

$$
\sum_{m \geq 1}\left\|h_{m}^{\varepsilon}\right\|_{L^{1}\left(Y^{\prime}\right)}=\sum_{m \geq 1} \int_{Y^{\prime}}\left|\widehat{u_{m}^{\varepsilon}}\left(\frac{\eta}{\varepsilon}\right)\right|^{2} d \eta=\left\|u^{\varepsilon}\right\|_{L^{2}\left(\mathbb{R}^{N}\right)}^{2}=1 .
$$

Each $h_{m}^{\varepsilon}$ is therefore bounded in $L^{1}\left(Y^{\prime}\right)$ and the sum of their norms is equal to 1 . Up to a subsequence, there exists a family of limit Radon measures $\left\{\nu_{m}(\eta)\right\}_{m \geq 1}$ such that each $h_{m}^{\varepsilon}$ converges to $\nu_{m}$ in the sense of vague measures. Of course, the limit measures are all non-negative, but they may well be zero. We can call these Bloch-limiting measures. Let us show that they satisfy

$$
\sum_{m \geq 1} \int_{Y^{\prime}} d \nu_{m}(\eta)=1
$$

which would prove that at least one of them is not identically zero. To prove (44), we use the following discrete version of the classical dominated convergence theorem.

Lemma 3.6. For each $\varepsilon>0$, let $\left\{a_{m}^{\varepsilon}\right\}_{m}$ be a sequence of real numbers such that the series $\sum_{m \geq 1} a_{m}^{\varepsilon}$ is convergent. Assume that the following alternative couple of conditions hold:

$$
\text { (i) } \forall m \geq 1, \exists a_{m} \in \mathbb{R}^{N} ; a_{m}^{\varepsilon} \longrightarrow a_{m} \text {, as } \varepsilon \rightarrow 0 \text {, }
$$




$$
\text { (ii) }\left\{\begin{array}{l}
\exists \text { a convergent series } \sum_{m \geq 1} \zeta_{m} ;\left|a_{m}^{\varepsilon}\right| \leq \zeta_{m} \quad \forall m \text {, or } \\
\forall \delta>0, \exists \text { a rank } m_{\delta} \geq 1 ; \sum_{m \geq m_{\delta}} a_{m}^{\varepsilon} \leq \delta \quad \forall \varepsilon .
\end{array}\right.
$$

Then the series $\sum_{m \geq 1} a_{m}^{\varepsilon}$ converges as $\varepsilon$ goes to zero and its limit is the series of the limits of its general terms, i.e., $\sum_{m \geq 1} a_{m}$.

Let us apply this lemma to the sequence $a_{m}^{\varepsilon}=\int_{Y}, h_{m}^{\varepsilon}(\eta) d \eta$. First, for each fixed $m$ we have

$$
\lim _{\varepsilon \rightarrow 0} \int_{Y^{\prime}} h_{m}^{\varepsilon}(\eta) d \eta=\int_{Y^{\prime}} d \nu_{m}(\eta)
$$

Next, let us assume that the second condition in (ii) is not satisfied. Then there exists $\delta>0$, a subsequence, still denoted by $\varepsilon$, and a sequence of integers $m(\varepsilon)$, going to $+\infty$, such that

$$
\sum_{m \geq m(\varepsilon)} \int_{Y} h_{m}^{\varepsilon}(\eta) d \eta>\delta
$$

Thanks to the boundedness of $\varepsilon \nabla u^{\varepsilon}$, by Parseval's Identity we have

$$
M \geq \varepsilon^{2}\left\|\nabla u_{\varepsilon}\right\|_{L^{2}\left(\mathbb{R}^{N}\right)}^{2} \geq \frac{1}{\beta} \sum_{m \geq 1} \int_{Y^{\prime}} \lambda_{m}(\eta) h_{m}^{\varepsilon}(\eta) d \eta \geq \frac{\delta}{\beta} \min _{\eta \in Y^{\prime}} \lambda_{m}(\eta),
$$

where $\beta=\max _{k, \ell}\left\|a_{k, \ell}\right\|_{L^{\infty}(Y)}$. But, this is a contradiction, since for any $\eta \in Y^{\prime}$

$$
\lim _{m \rightarrow+\infty} \lambda_{m}(\eta)=+\infty
$$

This completes the proof of (44).

Since the test function $\psi$ and the Bloch eigenvalues $\lambda_{m}$ are continuous in $\eta$, again with the help of Lemma 3.6 it is easily seen that one can pass to the limit in the relation (43):

$$
\sum_{m \geq 1} \int_{\dot{Y}^{\prime}} \psi_{m}(\eta)\left(\lambda_{m}(\eta)-\lambda\right) d \nu_{m}(\eta)=0
$$

Since at least one of the Bloch limiting measures $\nu_{m}$ is not trivial, there exists an energy level $m \geq 1$ and a Bloch frequency $\eta$ such that

$$
\lambda=\lambda_{m}(\eta)
$$

which finishes the proof of Theorem 1.5.

\section{Acknowledgments}

This text is a summary of the Lecture given by the second of the authors at the 29ème Congrès National d'Analyse Numérique (CANum'97) organized at Domain d'Imbours à Larnas en Ardèche (FRANCE) by the Equipe d'Analyse Numérique de l'Université Jean Monnet Saint Etienne. I would like to thank professors M. Ahues and C. Carasso very warmly for inviting me to give this Plenary Conference.

The authors acknowledge the support of the French Government through its Scientific Committee ECOS. The second author is also partially supported by the Chilean Programme of Presidential Chairs in Sciences and by Fondecyt under grant № 197 0734. The third author acknowledges the support received from IFCPAR, New Delhi. 


\section{References}

[1] F. Aguirre \& C. ConCA, (1988) Eigenfrequencies of a tube bundle immersed in a fluid, Appl. Math. Optim. 18, 1-38.

[2] G. Allaire, Homogenization and two-scale convergence, SIAM J. Math. Anal. 23, (1992), 1482-1518.

[3] G. Allaire \& C. ConCA, Bloch-wave homogenization and spectral asymptotic analysis, J. Math. Pures Appl. (1997), (submitted paper).

[4] G. Allaire \& C. ConCA, Boundary layers in the homogenization of a spectral problem in fluid-solid structures, SIAM J. Math. Anal., (1997), (in press).

[5] G. Allaire \& C. ConCa, Bloch wave homogenization for a spectral problem in fluidsolid structures, Arch. Rational Mech. Anal. 135(3), (1996), 197-257.

[6] G. Allaire \& C. Conca, Analyse asymptotique spectrale de l'équation des ondes. Homogénéisation par ondes de Bloch, C. R. Acad. Sci. Paris Sér. I Math. t.321, (1995), 293-298.

[7] G. Allaire \& C. Conca, Analyse asymptotique spectrale de l'équation des ondes. Complétude du spectre de Bloch, C. R. Acad. Sci. Paris Sér. I Math. t.321, (1995), $557-562$.

[8] A. Bensoussan, J.L. Lions \& G. Papanicolaou, Asymptotic Analysis in Periodic Structures, North-Holland, Amsterdam, 1978.

[9] F. BLoch, Über die Quantenmechanik der Electronen in Kristallgittern, Z. Phys. 52, (1928), 555-600.

[10] L. Boccardo \& P. Marcellini, Sulla convergenza delle soluzioni di disequazioni variazionali, Ann. Mat. Pura Appl. 4, (1977), 137-159.

[11] C. Castro \& E. Zuazua, Une remarque sur l'analyse asymptotique spectrale en homogénéisation, C. R. Acad. Sci. Paris Sér. I Math. t.322, (1996), 1043-1048.

[12] C. Conca, J. Planchard \& M. Vanninathan, Fluids and Periodic Structures, Collection RAM 38, J. Wiley \& Sons/Masson, New York, Paris, 1995.

[13] C. Conca, J. Planchard \& M. Vanninathan, Limiting behaviour of a spectral problem in fluid-solid structures, Asymp. Anal. 6, (1993), 365-389.

[14] C. Conca \& M. Vanninathan, Homogenization of periodic structures via Bloch decomposition, SIAM J. Appl. Math. 57, (1997), (in press).

[15] C. Conca \& M. Vanninathan, A spectral problem arising in fluid-solid structures, Comput. Methods Appl. Mech. Engrg. 69, (1988), 215-242.

[16] C. Conca, J. Planchard, B. Thomas \& M. Vanninathan, Problèmes Mathématiques en Couplage Fluide-Structure. Applications aux Faisceaux Tubulaires, Eyrolles, Paris, 1994.

[17] A. Figotin \& P. Kuchment, Band-gap structure of spectra of periodic dielectric and accoustic media. I, scalar model, SIAM J. Appl. Math. 56, (1996), 68-88.

[18] G. Floquet, Sur les équations différentielles linéaires à coefficients périodiques, Ann. Ecole Norm. Sér. 2 12, (1883), 47-89.

[19] I.M. GELFAnd, Expansion in series of eigenfunctions of an equation with periodic coefficients, Dokl. Akad. Nauk SSSR 73, (1950), 1117-1120.

[20] P. GÉrard, Mesures semi-classiques et ondes de Bloch, in Séminaire Équations aux Dérivées Partielles 1990-1991 16, École Polytechnique, Palaiseau, 1991. 
[21] S. Kesavan, Homogenization of elliptic eigenvalue problems, I and II, Appl. Math. Optim. 5, (1979), 153-167 \& 197-216.

[22] P.L. Lions \& T. Paul, Sur les mesures de Wigner, Revista Math. Iberoamer. 9, (1993), 553-618.

[23] P.A. Markowich, N.J. Mauser \& F. Poupaud, A Wigner function approach to semiclassical limits: electrons in a periodic potential, J. Math. Phys. 35, (1994), 10661094.

[24] R. Morgan \& I. BABUŠKa, An approach for constructing families of homogenized equations for periodic media I and II, SIAM J. Math. Anal. 2, (1991), 1-15 \& 16-33.

[25] F. Murat, H-Convergence, Séminaire d'Analyse Fonctionnelle et Numérique de l'Université d'Alger, mimeographed notes. English translation (1997), in Topics in the Mathematical Modelling of Composite Materials, A. CHERKAEV and R. KOHN eds., Series Progress in Nonlinear Differential Equations and their Applications 31, Birkhäuser Verlag, Boston, 1977-78.

[26] G. Nguetseng, A general convergence result for a functional related to the theory of homogenization, SIAM J. Math. Anal. 20, (1989), 608-623.

[27] F. Odeh \& J.B. Keller, Partial differential equations with periodic coefficients and Bloch waves in crystals, J. Math. Phys. 5, (1964), 1499-1504.

[28] O.A. Oleinik, A.S. Shamaev \& G.A. Yosifian, On the limiting behaviour of a sequence of operators defined in different Hilbert's spaces, Upsekhi Math. Nauk. 44, (1989), 157-158.

[29] J. Planchard, Global behaviour of large elastic tube-bundles immersed in a fluid, Comput. Mech. 2, (1987), 105-118.

[30] J. Planchard, Eigenfrequencies of a tube-bundle placed in a confined fluid, Comput. Methods Appl. Mech. Engrg. 30, (1982), 75-93.

[31] M. Reed \& B. Simon, Methods of Modern Mathematical Physics, I. Functional Analysis, II. Fourier Analysis and Self-Adjointness, III. Scattering Theory, IV. Analysis of Operators, Academic Press, New York, 1972-78.

[32] E. Sanchez-Palencia, Non-Homogeneous Media and Vibration Theory, Lecture Notes in Physics 127, Springer-Verlag, Berlin, 1980.

[33] J. Sanchez-Hubert \& E. Sanchez-Palencia, Vibration and Coupling of Continuous Systems. Asymptotic Methods, Springer-Verlag, Berlin, 1989.

[34] F. Santosa \& W.W. Symes, A dispersive effective medium for wave propagation in periodic composites, SIAM J. Appl. Math. 51 (4), (1991), 984-1005.

[35] L. TARTAR, H-measures, a new approach for studying homogenization, oscillations and concentration effects in partial differential equations, Proc. Roy. Soc. Edinburgh 115 A, (1990), 193-230.

[36] L. Tartar, Problèmes d'Homogénéisation dans les Équations aux Dérivées Partielles, Cours Peccot au Collège de France, partially written in F. MURAT [25], 1977.

[37] M. Vanninathan, Fourier Approach to Homogenization, Invited Lecture at the International Conference on Nonlinear Differential Equations and Applications, Bangalore, India, 1996.

[38] M. Vanninathan, Homogenization and eigenvalue problems in perforated domains, Proc. Indian Acad. Sci. Math. Sci. 90, (1981), 239-271.

[39] C. Wilcox, Theory of Bloch waves, J. Anal. Math. 33, (1978), 146-167. 